\title{
Colchicum pusillum Extract Induced Apoptosis in Colo-741 Metastatic Colon Cancer Cells via Extrinsic Pathway $^{\dagger}$
}

\author{
Eda Becer 1, Duygu Yiğit Hanoğlu 2, Günsu Soykut 3,*, Azmi Hanoğlu 2, Seda Vatansever 4 , \\ Dudu Özkum Yavuz ${ }^{5}$, Filiz Meriçli ${ }^{5}$ and Ali Hikmet Meriçli ${ }^{5}$
}

1 Department of Biochemistry, Faculty of Pharmacy, Near East University, 99138 Nicosia, Mersin 10, Turkey; edabecer@hotmail.com

2 Department of Pharmaceutical Botany, Faculty of Pharmacy, Near East University, 99138 Nicosia, Mersin 10, Turkey; duygu.yigithanoglu@neu.edu.tr (D.Y.H.); azmi.hanoglu@neu.edu.tr (A.H.)

3 Department of Nutrition and Dietetics, Faculty of Health Sciences, Near East University, 99138 Nicosia, Mersin 10, Turkey

4 Department of Histology and Embryology, Faculty of Medicine, Manisa Celal Bayar University, 45140 Manisa, Turkey; sedavatansever@yahoo.com

5 Department of Pharmacognosy-Phytotherapy, Faculty of Pharmacy, Near East University, 99138 Nicosia, Mersin 10, Turkey; dudu.ozkum@neu.edu.tr (D.Ö.Y.); filiz.mericli@neu.edu.tr (F.M.); ali.mericli@neu.edu.tr (A.H.M.)

* Correspondence: gunsusoykut@gmail.com

† Presented at the 2nd International Cell Death Research Congress, Izmir, Turkey, 1-4 November 2018.

Published: 5 December 2018

\begin{abstract}
Colchicum pusillum Sieber is a plant from Colchicaceae family that particularly rich in tropolonic alkaloids, colchicine. The aim of the study is to determine the apoptosis induction effect of Colchicum pusillum extract in Colo-741, metastatic colon cancer cells and if so, to determine by which apoptotic pathway. Colchicum pusillum was collected and extracted with ethanol. Apoptotic activities of Colchicum pusillum were investigated by immunocytochemistry using antibodies directed against to caspase-3, cytochrome-c and FasLigand (FasL) in Colo-741 cells. Immunocytochemical staining results stated that Colchicum pusillum extract at $20 \mu \mathrm{g} / \mathrm{mL}$ dose, increased caspase-3 and FasL activity significantly but was not able to activate cytochrome-c activity. The results suggested that Colchicum pusillum extract induced apoptosis in metastatic colon cancer cells via extrinsic apoptotic pathway.
\end{abstract}

Keywords: Colchicum pusillum; colon cancer; apoptosis

\section{Introduction}

Colchicum species, part of Colchicaceae family, are flowering plants known to exert medicical properties. There are more than Colchicum 100 species, three of them are being endemic to Cyprus. These are; Colchicum troodi Kotschy, Colchicum stevenii Kunth and Colchicum pusillum Sieber, respectively. Colchicum pusillum is being more widespread in North Cyprus [1]. Colchicum species contains alkaloids and mainly colchicine. Colchicine has been found to possess antitumoral and antiphlogistic properties. To this day, colchicine is still used in treatment of gout and many other diseases [2]. Colorectal cancer is the third most common cancer in the world and the fourth cancer with the highest mortality incidence [3]. Colon cancer is a disease caused by the formation of malignant cells in colon tissue [4]. The aim of the study is to determine the apoptosis induction effect 
of Colchicum pusillum Sieber extract in Colo-741, metastatic colon cancer cells and if so, to determine by which apoptotic pathway.

\section{Materials and Methods}

\subsection{Plant Material and Extraction}

The Extract was obtained from the bulbs of Colchicum pusillum Sieber (Colchicaceae) which were collected in North Cyprus. The plant material was registered at the Herbarium of Near East University, under the code NEUN 6892. Powdered plant material was extracted with Ethanol with Soxhlet Apparatus. Ethanol was evaporated with rotary evaporator and then dissolved in tertiary butanol-water in the ratio of 1:3. The extract was further dried in a freeze dryer for $36 \mathrm{~h}$ to yield $0.5 \mathrm{~g}$ of pure dried extract.

\subsection{Cell Line and Cell Culture}

Colo-741 (ECACC: 93052621), human colon adenocarcinoma cell line was used. The cells were maintained in RPMI-1640 medium (Biochrom, FG 1215, Berlin, Germany), with added $10 \%$ heat inactivated fetal bovine serum (FBS) (Capricorn Scientific, FBS-11B, Ebsdorfergrund, Germany), 1\% penicillin-streptomisin (Biochrom, A2213, Berlin, Germany) and 1\% glutamine (EMD Millipore, K0282, Darmstadt, Germany).

\subsection{Immunocytochemistry}

Cultured Colo-741 cells were assessed immunocytochemically for binding of antibodies against caspase-3, cytochrome-c and FasLigand (FasL). Colo741 cells were fixed with $4 \%$ paraformaldehyde in PBS at $4{ }^{\circ} \mathrm{C}$ for $30 \mathrm{~min}$. After Tween 20 (Sigma-Aldich, Darmstadt, Germany) was added for permeabilization for $15 \mathrm{~min}$, the cells were washed with PBS and endogenous peroxidase activity was quenched by incubation with $3 \% \mathrm{H}_{2} \mathrm{O}_{2}$ for $5 \mathrm{~min}$ at room temperature. After washing cells with PBS, primary antibodies caspase-3 (sc-7272, Santa Cruz Biotechnology, Inc., Dallas, TX, USA), cytochrome-c (sc-13156, Santa Cruz Biotechnology, Inc., Dallas, TX, USA) and FasL (sc-834 Santa Cruz Biotechnology, Inc., Dallas, TX, USA) were added and incubated overnight at $4{ }^{\circ} \mathrm{C}$. Biotinylated secondary antibody (Histostain-Plus, IHC Kit, HRP, 859043, Thermo Fischer, Frederick, MD, USA) was added and incubated for $30 \mathrm{~min}$ followed by PBS wash. In total, $100 \mu \mathrm{L}$ strepavidin-peroxidase complex was added to cultured cells. DAB was added and incubated for 5 min and washed with distilled water. Cells were counterstained with Mayer's hematoxylien for 5 min and mounted with mounting medium (Merck Millipore, 107961, Darmstadt, Germany). All specimens were examined using a light microscope (Olympus BX40, Tokyo, Japan). Staining of caspase-3, cytochrome-c and FasL was also graded semi quantitatively using the H-SCORE and was calculated with the following equation: $\mathrm{HSCORE}=\Sigma_{\Lambda}(\mathrm{i}+1)$.

\subsection{Statistical Analysis}

Results were expressed as mean \pm standard deviation (SD). The results were analyzed using GraphPad Prism 7 software. Differences among groups were analyzed statistically with Mann-Whitney test where appropriate. A $p$ value of $<0.05$ was regarded as statistically significant.

\section{Results}

\subsection{Cell Morphology}

Colo-741 cells are fibroblast-like cells that grow with typical fibroblast colony morphology after $48 \mathrm{~h}$ in culture. Colchicum pusillum extract treated group has shown changes in shape to oval than fibroblast-like structure and total number of cells was less than control group. 


\subsection{Immunocytochemical Evaluation}

Caspase-3 immunostaining was moderate to strong in Colo-741 cells treated with Colchicum pusillum extract (Table 1). The H-SCORE and immunoreactivity results were in parallel with immunostaining intensity, where H-SCORE for caspase-3 was significantly higher in extract treated cells treated when compared with control group $(p<0.05$, Table 2) (Figure 1A,B). Immunostaining intensity for cytochrome-c was moderate in cells treated with Colchicum pusillum extract (Table 1). Although, cytochrome-c immunoreactivity was higher in extract treated Colo-741 cells than in control group, the results were not statistically significant $(p>0.05$, Table 2$)$. FasL immunostaining intensity results showed extract exerted moderate to strong effect in metastatic colon cancer cells (Table 1) with similar patterns observed in immunoreactivity (Figure 1E,F). In addition, the H-SCORE results stated that FasL immunoreactivity was significantly higher in extract treated cells than control group $(p<0.05$, Table 2$)$.

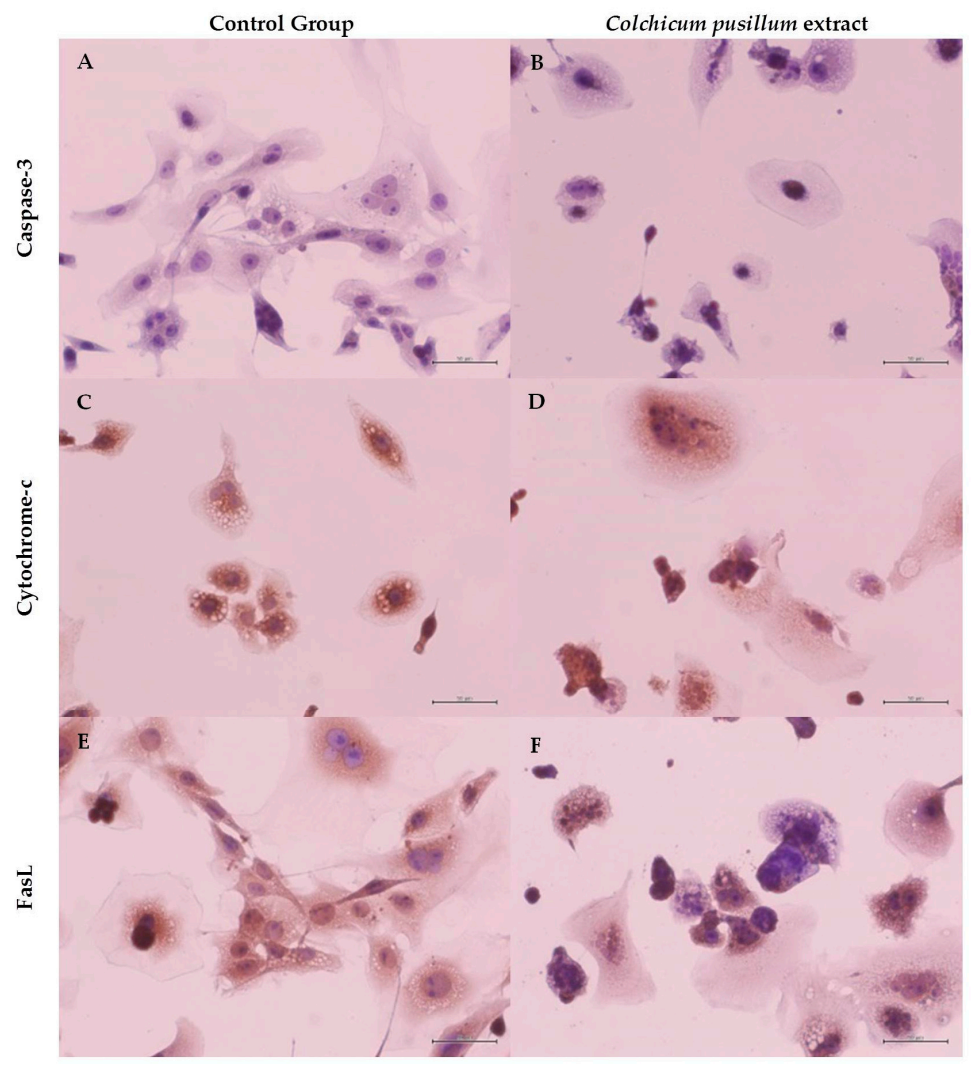

Figure 1. Immunoreactivity of caspase-3, cytochrome-c and FasL in Colo-741 control cells and Colchicum pusillum extract treated Colo-741 cells at $20 \mu \mathrm{g} / \mathrm{mL}$ concentration for $48 \mathrm{~h}$. Scale Bars $=20 \mu \mathrm{m}$.

Table 1. The intensity of caspase-3, cytochrome-c and FasL immunolabelling in Colo-741 cells treated with Colchicum pusillum extract at $20 \mu \mathrm{g} / \mathrm{mL}$ concentration for $48 \mathrm{~h}$.

\begin{tabular}{ccc}
\hline & Control Group & Colchicum pusillum Extract \\
\hline Caspase-3 & $+/++$ & $++/++$ \\
Cytochrome-c & $+/++$ & ++ \\
FasL & $+/++$ & $++/+++$ \\
\hline
\end{tabular}

Table 2. The H-SCORE of Caspase-3, cytochrome-c and FasL in Colo-741 cells treated with Colchicum pusillum extract at $20 \mu \mathrm{g} / \mathrm{mL}$ concentration for $48 \mathrm{~h}$.

\begin{tabular}{cccc}
\hline & Caspase-3 & Cytochrome-c & FasL \\
\hline Control group & $236.37 \pm 28.39$ & $221 \pm 6.42$ & $217.6 \pm 27.22$ \\
\hline Colchicum pusillum extract $^{3} 302.53 \pm 37.37^{\text {a }}$ & $269.45 \pm 49.22$ & $288.56 \pm 11.45^{\text {a }}$ \\
\hline
\end{tabular}

a The data was significant when compared with control group $(p<0.05)$. 


\section{Discussion}

In apoptosis process, presence of FasL and caspase- 8 show that apoptosis is triggered by extrinsic pathway whereas cytochrome-c, caspase- 9 and Apaf- 1 are indicators of intrinsic pathway [5]. Our results showed that immunoreactivity of cytochrome-c was not significant in extract treated Colo-741 cells. This states that the apoptosis is not triggered via intrinsic pathway. However, a study by Huang et al. (2015) stated that colchicine induced apoptosis in HT-29 colon cancer cells via mitochondria-dependent pathway by decreasing mitochondrial membrane potential and possibly increasing cytochrome-c leakage from mitochondria [6]. The immunoreactivity of FasL had shown a significant increase in extract treated Colo-741 cells when compared with control group. This indicates that, FasL is activated with the help of extract. Caspase-3 is known as executioner caspase. Its immunoreactivity was significantly higher in extract treated Colo-741 cells indicating that last step of apoptotic pathway had been reached. These results indicates that apoptosis was triggered in metastatic colon adenocarcinoma cell lines via extrinsic apoptotic pathway. Colchicine is known to have anti-mitotic activity through microtubule depolymerisation. According to some studies colchicine also exerts anti-cancer effect and can be used as a chemotherapeutic agent [6,7]. Main mechanism behind colchicine showing anti-cancer properties is thought to be via direct colchicine tubulin interaction, by disturbing microtubule aggregation $[6,8]$. In conclusion, our results showed that Colchicum pusillum extract was able to activate caspase- 3 and therefore apoptosis by triggering extrinsic apoptotic pathway via increasing FasL activity. All of these studies suggest that Colchicum species have great potential to be used as anti-cancer drug. However, further detailed mechanistic studies should be done in order to suggest Colchicum pusillum plant as a chemotherapeutic agent.

\section{References}

1. Viney, D.E. An Illustrated Flora of North Cyprus, Vol 1; Koeltz Scientific Books: Koenigstein, Germany, 1994; p. 697.

2. Le, H.C. The pharmacology and therapeutic aspects of colchicine. Alkaloids 2000, 53, 288-352.

3. Global Cancer Observatory (GCO). Cancer Today. 2018. Available online: https://gco.iarc.fr/today/onlineanalysis-multi-bars?mode $=$ cancer\&mode $\_$population $=$continents\&population $=900 \&$ sex $=0 \&$ cancer $=29 \&$ type $=$ $0 \&$ statistic $=0 \&$ prevalence $=0 \&$ color_palette $=$ default $($ accessed on 31 May 2018).

4. National Cancer Institute (NIH). Colorectal Cancer-Health Professional Version. 2018. Available online: https://www.cancer.gov/types/colorectal/hp (accessed on 31 May 2018).

5. Huerta, S.; Goulet, E.J.; Livingston, E.H. Colon cancer and apoptosis. Am. J. Surg. 2006, 191, 517-526, doi:10.1016/j.amjsurg.2005.11.009.

6. Huang, Z.; Xu, Y.; Peng, W. Colchicine induces apoptosis in HT-29 human colon cancer cells via the AKT and c-Jun N-terminal kinase signaling pathways. Mol. Med. Rep. 2015, 12, 5939-5944, doi:0.3892/mmr.2015.4222.

7. Lin, Z.Y.; Wu, C.C.; Chuang, Y.H.; Chuang, W.L. Anti-cancer mechanisms of clinically acceptable colchicine concentrations on hepatocellular carcinoma. Life Sci. 2013, 93, 323-328, doi:10.1016/j.lfs.2013.07.002.

8. Lin, Z.Y.; Kuo, C.H.; Wu, D.C.; Chuang, W.L. Anticancer effects of clinically acceptable colchicine concentrations on human gastric cancer cell lines. Kaohsiung J. Med. Sci. 2016, 32, 68-73, doi:10.1016/j.kjms.2015.12.006. 